\title{
Middle and lower esophagectomy preceded by hand-assisted laparoscopic transhiatal approach for distal esophageal cancer
}

\author{
ATSUSHI SHIOZAKI, HITOSHI FUJIWARA, HIROTAKA KONISHI, RYO MORIMURA, SHUHEI KOMATSU, \\ YASUTOSHI MURAYAMA, YOSHIAKI KURIU, HISASHI IKOMA, TAKESHI KUBOTA, \\ MASAYOSHI NAKANISHI, DAISUKE ICHIKAWA, KAZUMA OKAMOTO, \\ CHOUHEI SAKAKURA and EIGO OTSUJI
}

Division of Digestive Surgery, Department of Surgery, Kyoto Prefectural University of Medicine, Kyoto 602-8566, Japan

Received April 26, 2013; Accepted October 10, 2013

DOI: $10.3892 /$ mco.2013.201

\begin{abstract}
Respiratory morbidity is the most frequent complication following an esophagectomy. This study was designed to determine the efficacy of middle and lower esophagectomies preceded by the hand-assisted laparoscopic transhiatal approach (LTHA) regarding the perioperative outcomes of distal esophageal cancer. The esophageal hiatus was opened and carbon dioxide was introduced into the mediastinum. Dissection of the distal esophagus was performed up to the level of the tracheal bifurcation. En bloc dissection of the posterior mediastinal lymph nodes was performed using the LTHA. Subsequently, a small thoracotomy $(10 \mathrm{~cm})$ was performed to divide the thoracic esophagus and allow middle mediastinal lymphadenectomy. Finally, reconstruction via the posterior mediastinal route with a gastric tube and anastomosis in the thoracic cavity were performed using a circular stapler. The treatment outcomes of 10 patients who underwent LTHA-preceded middle and lower esophagectomy were compared to those of 11 patients treated without prior LTHA (thoracotomy, $20 \mathrm{~cm}$ ). The total operative time, the duration of one-lung ventilation and total operative blood loss were significantly decreased in the LTHA group. The number of resected lymph nodes did not differ significantly between the two groups. Postoperative respiratory complications occurred in $10.0 \%$ of patients treated with, and $36.3 \%$ of those treated without LTHA. The extubation time following surgery, the duration of thoracic drainage and postoperative hospital stay were significantly decreased by this method. In conclusion, middle and lower esophagectomies preceded by LTHA provides a good surgical view of the posterior mediastinum, markedly shortens the duration of one-lung ventilation and improves the perioperative outcome.
\end{abstract}

Correspondence to: Dr Atsushi Shiozaki, Division of Digestive Surgery, Department of Surgery, Kyoto Prefectural University of Medicine, 465 Kajii-cho, Kamigyo-ku, Kyoto 602-8566, Japan E-mail: shiozaki@koto.kpu-m.ac.jp

Key words: laparoscopic transhiatal approach, distal esophageal cancer

\section{Introduction}

Esophagectomies is associated with significant morbidity and mortality, although recent advances in surgical and postoperative management techniques have improved the treatment outcome (1). Respiratory morbidity, in particular, remains the most common serious complication following esophagectomy and a number of studies demonstrated a respiratory complication rate of $20 \%$ (2-5). Since the duration of one-lung ventilation is known to affect postoperative immune reactions and cause respiratory complications, it is crucial to reduce the intrathoracic operative time $(6,7)$.

We first performed an esophagectomy preceded by the laparoscopic transhiatal approach (LTHA) for patients with esophageal cancer in 2009 (8-10). With this method, carbon dioxide is introduced into the mediastinum from the abdominal side of the diaphragm and middle and lower mediastinal operations may be performed via a transhiatal approach. The main advantages of this method are that the thoracic procedures performed via right thoracotomy may be simplified and the duration of one-lung ventilation may be shortened. In addition, a good surgical view of the lower mediastinum is obtained and the quality of the mediastinal surgery may be improved. By December, 2012, a total of 121 patients with esophageal tumors had undergone LTHA during a variety of esophageal surgical procedures, including subtotal esophagectomy, middle and lower esophagectomy and tumor resection (8-11).

In our previous study, we demonstrated the efficacy of LTHA preceding subtotal esophagectomy with gastric tube reconstruction via a retrosternal route, with regard to perioperative outcomes (8). In this study, we aimed to analyze the perioperative treatment outcomes of patients with distal esophageal cancer who underwent middle and lower esophagectomy preceded by hand-assisted LTHA and gastric tube reconstruction via the posterior mediastinal route (anastomosis in the thoracic cavity). Our results revealed that our method markedly shortened the duration of one-lung ventilation and decreased intraoperative blood loss. Furthermore, this method improved postoperative care by decreasing the extubation time following surgery, the duration of thoracic drainage and the length of the postoperative hospital stay. 
A

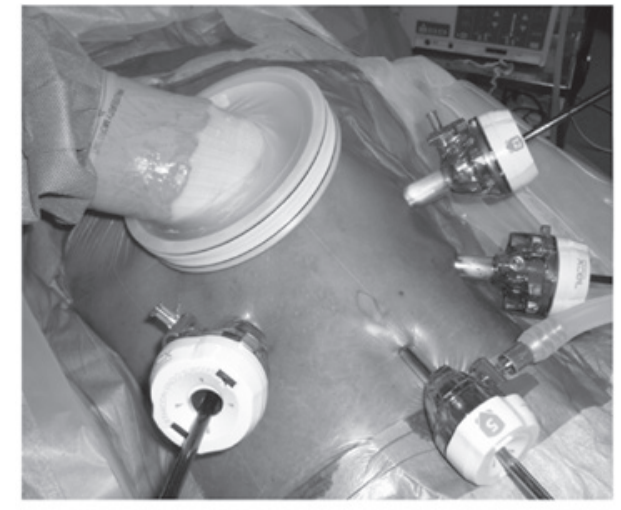

C

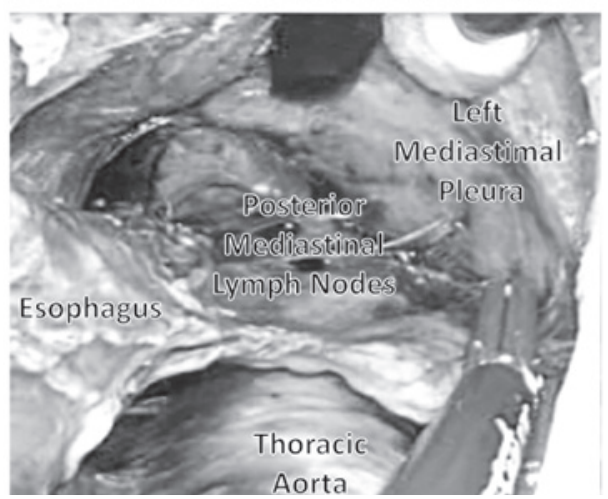

B

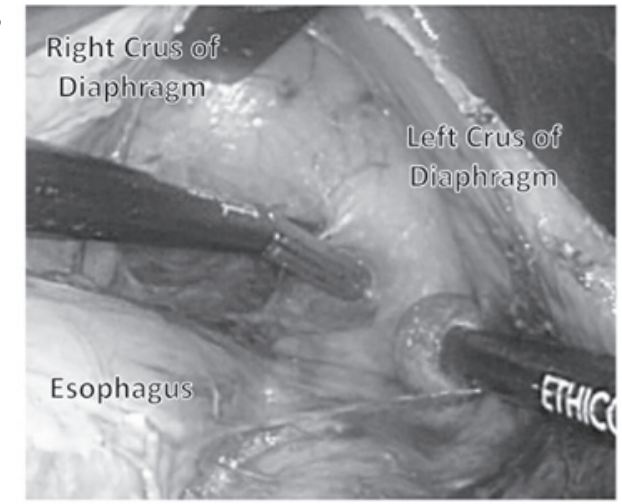

D

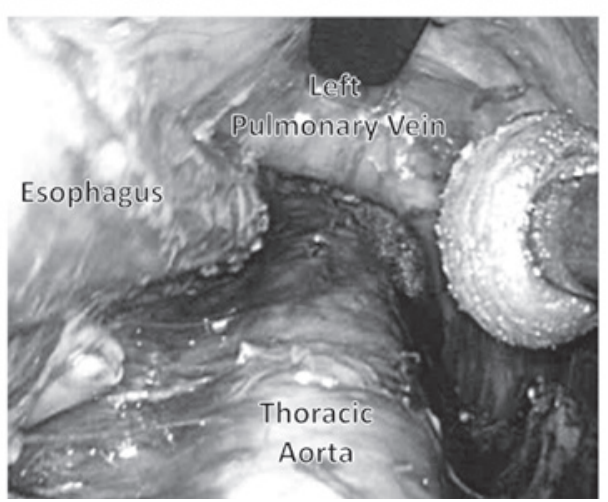

Figure 1. (A) Intraoperative view of the trocars and incision locations on the abdomen. A lap disc (regular) was placed in the upper abdomen. Three 12-mm ports were inserted, one in each flank and one in the left hypochondrium; one 5-mm port for the videoscope was inserted in the lower abdomen. (B) The esophageal hiatus was divided and carbon dioxide was introduced into the mediastinum. Dissection of the anterior sides of the posterior mediastinal lymph nodes was performed with the EnSeal device. (C) The anterior and posterior sides of the posterior mediastinal lymph nodes were resected: while lifting up these lymph nodes like a membrane, they were cut along the borderline of the left mediastinal pleura. (D) The posterior mediastinal lymph nodes (thoracic paraaortic and left pulmonary ligament lymph nodes) were dissected en bloc.

\section{Patients and methods}

Surgical procedure. An abdominal operation was performed using hand-assisted laparoscopic surgery (HALS). Subsequently, middle and lower mediastinal operations were performed using LTHA. The patients were placed in a supine position on the operating table. An upper abdominal incision $(70 \mathrm{~mm})$ was performed and a Lap Disc (regular) (Ethicon Endo-Surgery, Cincinnati, OH, USA) was placed (Fig. 1A). Three 12-mm ports were inserted, one in each flank and one in the left hypochondrium; one 5-mm port for the flexible laparoscope was inserted into the lower abdomen (Fig. 1A). The operator stood on the right side of the patient and inserted the Lap Disc with their left hand. The 12-mm port in the right flank was mainly used for the surgery. The assistant stood on the left side of the patient and the ports in the left abdomen were used to provide assistance. The scopist stood near the patient's groin. Carbon dioxide was introduced into the intra-abdominal space and the pneumoperitoneum pressure was controlled at $10 \mathrm{mmHg}(8-11)$.

The operator lifted up the stomach with their left hand and the greater omentum, left gastroepiploic vessels and gastrosplenic ligament were divided using the EnSeal device (Ethicon Endo-Surgery). Subsequently, the esophageal hiatus was opened and carbon dioxide was introduced into the mediastinum. The assistant inserted an Endo Retract (Autosuture Norwalk, CT, USA) and a blunt tip dissector through the ports on the left side and the working space in the mediastinum was secured with these two devices and $10 \mathrm{mmHg}$ of pneumomediastinum pressure.
Dissection of the anterior and left side of the distal esophagus up to the level of the tracheal bifurcation was performed with the EnSeal device and the blunt tip dissector. Using this approach, dissection of the anterior sides of the posterior mediastinal lymph nodes was easily performed (Fig. 1B) (9).

Subsequently, the adventitia of the thoracic aorta was exposed at the level of the crura of the diaphragm, followed by dissection of the anterior side of the thoracic aorta to the cranial side. The roots of the proper esophageal arteries were confirmed and divided using the EnSeal device. Following these procedures, the anterior and posterior sides of the posterior mediastinal lymph nodes, including the thoracic paraaortic and left pulmonary ligament lymph nodes, were dissected. While lifting these lymph nodes like a membrane, they were cut along the borderline of the left mediastinal pleura (Fig. 1C). In this manner, the posterior mediastinal lymph nodes were dissected en bloc (Fig. 1D) $(8,9)$.

Following the dissection of posterior mediastinal lymph nodes, the adventitia of the thoracic aorta and the crura of the diaphragm were exposed. Therefore, the appropriate layer for the dissection of the celiac lymph nodes was clearly identified and the dissection of the posterior mediastinal lymph nodes was extended towards the caudal side from the crura of the diaphragm to the celiac artery (Fig. 2A). The lymph nodes in the esophageal hiatus of the diaphragm, the infradiaphragmatic lymph nodes and the lymph nodes along the celiac artery were dissected en bloc from the left side approach. Subsequently, the left gastric vessels were exposed from the 
A

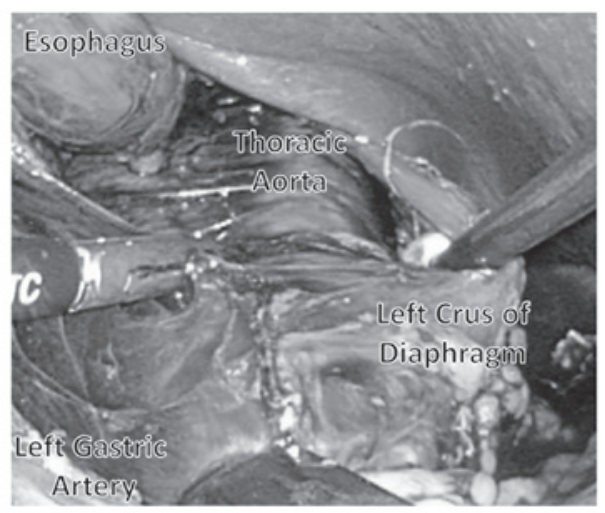

C

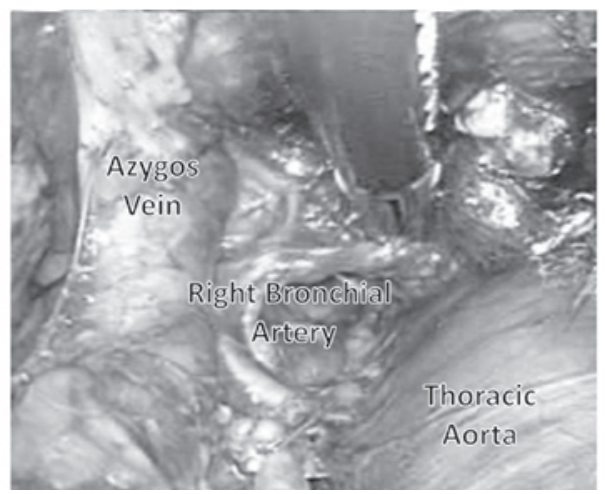

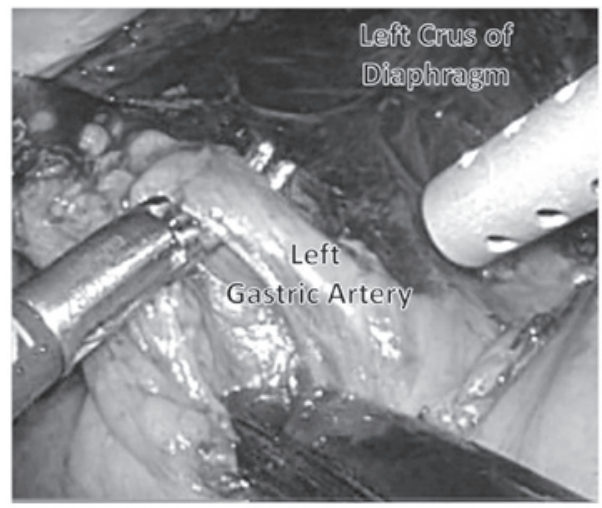

D

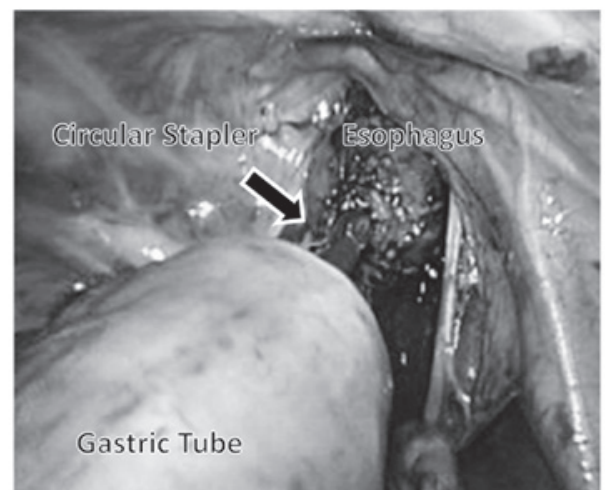

Figure 2. (A) The dissection of the posterior mediastinal lymph nodes was extended towards the caudal side from the crura of the diaphragm to the celiac artery. (B) The left gastric artery was exposed from the left side and the lymph nodes along the left gastric artery were dissected. (C) An incision was made in the right mediastinal pleura and was extended to the level of the arch of the azygos vein. (D) Reconstruction via the posterior mediastinal route with a gastric tube and anastomosis in the thoracic cavity were performed using a circular stapler (CDH25) via the thoracic approach.

left side, clipped and divided and the lymph nodes along the left gastric artery were dissected (Fig. 2B). Thus, the posterior mediastinal and celiac lymph nodes that were located around the border between the thoracic and the abdominal cavity were continuously dissected with the LTHA.

Dissection of the posterior and right sides of the distal esophagus was subsequently performed and an incision was made in the right mediastinal pleura to allow ablation. The right mediastinal pleural incision was then extended to the level of the arch of the azygos vein (Fig. 2C). Thus, the middle and lower thoracic esophagus was completely detached from the surrounding tissue.

Subsequently, in the left semilateral-decubitus position, the operating table was rotated to the left, a small right thoracotomy was performed $(\sim 10 \mathrm{~cm})$ and a rib retractor was used to maintain the small surgical field. The middle mediastinal lymph nodes, including subcarinal and bilateral main bronchial lymph nodes, were resected via the thoracic approach. Following division of the azygos vein, the upper thoracic esophagus was divided.

The operating table was then rotated to the right and the middle and lower thoracic esophagus was extracted via an upper abdominal incision. Finally, reconstruction via the posterior mediastinal route with a gastric tube and anastomosis in the thoracic cavity were performed using a CDH25 circular stapler (Ethicon Endo-Surgery) via the thoracic approach (Fig. 2D).

Patients. We started to routinely perform LTHA-preceded middle and lower esophagectomies in patients with distal esophageal cancer in December, 2009. Between January, 2005 and December, 2012, a total of 21 patients with esophageal cancer underwent middle and lower esophagectomy combined with lymph node dissection and gastric tube reconstruction via the posterior mediastinal route (anastomosis in the thoracic cavity) at the Division of Digestive Surgery, Department of Surgery, Kyoto Prefectural University of Medicine. The patients were retrospectively divided into two groups according to the operative method, with and without LTHA (10 patients from December, 2009 to December, 2012; and 11 patients from January, 2005 to November, 2009, respectively). The two groups were compared with respect to the perioperative treatment outcome. The operative indication was identical in the two groups, namely clinical T1-3, N0-3, M0 esophageal cancer, staged according to the International Union Against Cancer TNM classification of malignant tumours, 7th edition (12). All patients were treated by two highly skilled surgeons and informed consent was obtained from each participant.

Surgical procedure without LTHA. For the 11 patients who underwent middle and lower esophagectomy without LTHA the procedure was as follows: the patient was placed in the left semilateral-decubitus position, the operating table was rotated to the right and abdominal surgery was performed first via laparotomy $(20 \mathrm{~cm})$. The operating table was then rotated to the left and thoracic surgery (including middle and lower mediastinal operation) was performed via thoracotomy $(\sim 20 \mathrm{~cm})$. Finally, reconstruction via the posterior mediastinal route with a gastric tube and anastomosis in the thoracic 
cavity were performed using a $\mathrm{CDH} 25$ circular stapler, via the thoracic approach.

All the patients underwent two-field lymphadenectomy. A total of 6 patients ( 3 patients with and 3 without LTHA) received preoperative chemotherapy, including 2 courses of cisplatin $\left(80 \mathrm{mg} / \mathrm{m}^{2} /\right.$ day on day 1) plus 5 -fluorouracil (800 mg/m²/day on days $1-5)(13)$.

Clinicopathological characteristics. To compare the backgrounds of the two groups, we analyzed their clinicopathological characteristics, such as age, gender, primary tumor location, histological type, TNM category and pathological stage. Histopathological examinations were performed on the primary lesions and all the dissected lymph nodes using serial sections. The histopathological diagnoses were confirmed by experienced pathologists. The TNM category and pathological stage were classified according to the pTNM pathological classification (12). The effects of preoperative comorbidities were analyzed using the Charlson comorbidity index (14).

Perioperative factors. To determine the efficacy of LTHA, the two groups were compared with respect to several perioperative factors, such as total operative time, duration of one-lung ventilation, operative blood loss, number of resected lymph nodes, duration of management by respirator, frequency of postoperative respiratory complications, number of white blood cells, serum C-reactive protein (CRP) level, duration of thoracic drainage, frequency of anastomotic leakage and postoperative hospital stay. The number of peripheral white blood cells and the serum CRP level were measured on days 2 and 7 following surgery. According to our criteria, the thoracic drainage tube was removed when the amount of thoracic discharge was reduced to $<150 \mathrm{ml} /$ day. Postoperative respiratory complications were defined as those involving major respiratory insufficiency; such as, a need for reintubation or severe pneumonia.

Statistical analysis. Statistical analysis was performed using the Student's t-test and Fisher's exact test. $\mathrm{P}<0.05$ was considered to indicate a statistically significant difference. All analyses were performed using JMP statistical software, version 5 (SAS Institute Inc., Cary, NC, USA).

\section{Results}

Patients. A total of 21 patients with esophageal cancer who underwent middle and lower esophagectomy combined with gastric tube reconstruction in the thoracic cavity were divided into two groups according to the operative procedure, such as, with and without LTHA (10 and 11 patients, respectively). Although the percentage of female patients was significantly higher in the LTHA group, there were no significant differences in the other clinicopathological parameters (age, primary tumor location, histological type, TNM category, pathological stage or Charlson comorbidity index) between the two groups (Table I). Two patients (one with and one without LTHA) were pathologically diagnosed as pT4 (invasion of the diaphragm).

Intraoperative factors. We performed a comparison of the intraoperative factors between the two groups. The total operative
Table I. Comparison of clinicopathological parameters of the patients who underwent middle and lower esophagectomy (anastomosis in the thoracic cavity) with and without preceding laparoscopic transhiatal approach (LTHA).

\begin{tabular}{|c|c|c|c|}
\hline \multirow[b]{2}{*}{ Variables } & \multicolumn{2}{|c|}{ LTHA } & \multirow[b]{2}{*}{ P-value } \\
\hline & With & Without & \\
\hline Age, years $($ mean \pm SEM $)$ & $66.1 \pm 3.2$ & $67.4 \pm 3.2$ & 0.781 \\
\hline \multicolumn{4}{|l|}{ Gender } \\
\hline Male & 6 & 11 & $0.035^{\mathrm{a}}$ \\
\hline Female & 4 & 0 & \\
\hline \multicolumn{4}{|l|}{ Location of primary tumor } \\
\hline Distal thoracic esophagus & 5 & 8 & 0.387 \\
\hline Abdominal esophagus & 5 & 3 & \\
\hline \multicolumn{4}{|l|}{ Histological type } \\
\hline $\mathrm{SCC}$ & 5 & 8 & 0.387 \\
\hline Other & 5 & 3 & \\
\hline \multicolumn{4}{|l|}{ pT category } \\
\hline pT0-1 & 3 & 3 & 1.000 \\
\hline pT2-4 & 7 & 8 & \\
\hline \multicolumn{4}{|l|}{ pN category } \\
\hline pNO & 4 & 6 & 0.670 \\
\hline pN1-3 & 6 & 5 & \\
\hline \multicolumn{4}{|l|}{ Pathological stage } \\
\hline 0 -II & 4 & 6 & 0.670 \\
\hline III-IV & 6 & 5 & \\
\hline $\begin{array}{l}\text { Charlson comorbidity index } \\
(\text { mean } \pm \text { SEM) }\end{array}$ & $2.0 \pm 0.1$ & $2.2 \pm 0.1$ & 0.172 \\
\hline
\end{tabular}

SEM, standard error of the mean. ${ }^{\mathrm{a}} \mathrm{P}<0.05$ was considered to indicate a statistically significant difference. SCC, squamous cell carcinoma.

time was significantly shorter in the group treated with compared to that in the group treated without LTHA (mean \pm SEM: $304.3 \pm 23.3$ vs. $485.0 \pm 22.3 \mathrm{~min}$, respectively) (Table II). The duration of one-lung ventilation was significantly shorter in patients treated with compared to that in patients treated without LTHA (mean \pm SEM: $144.4 \pm 16.5$ vs. $212.5 \pm 16.0 \mathrm{~min}$, respectively) (Table II). The total operative blood loss was significantly reduced in patients treated with compared to those treated without LTHA (mean \pm SEM: $272.9 \pm 101.4$ vs. $567.7 \pm 98.6 \mathrm{ml}$, respectively) (Table II). The total number of resected lymph nodes did not differ significantly between the two groups $(24.3 \pm 3.6$ and $27.4 \pm 3.3$ with and without LTHA, respectively) (Table II). The total number of resected thoracic lymph nodes did not differ significantly between the two groups (5.3 \pm 1.9 and $8.7 \pm 1.9$ with and without LTHA, respectively). These results suggest that LTHA-preceded esophagectomy may be used to reduce the total operative time, the duration of one-lung ventilation and total operative blood loss, without compromising the quality of lymph node dissection.

Postoperative factors. We then performed a comparison of the postoperative factors between the two groups (Table III). 
Table II. Comparison of intraoperative factors between patients who underwent middle and lower esophagectomy (anastomosis in the thoracic cavity) with and without laparoscopic transhiatal approach (LTHA).

\begin{tabular}{lccr}
\hline \multirow{2}{*}{ Variables } & \multicolumn{2}{c}{ LTHA } & Without \\
\cline { 3 - 4 } & With & $485.0 \pm 22.3$ & $<0.001^{\text {a }}$ \\
\hline Total operative time (min) & $304.3 \pm 23.3$ & $212.5 \pm 16.0$ & $0.008^{\mathrm{a}}$ \\
Duration of one-lung ventilation (min) & $144.4 \pm 16.5$ & $567.7 \pm 98.6$ & $0.049^{\mathrm{a}}$ \\
Total operative blood loss (ml) & $272.9 \pm 101.4$ & $27.4 \pm 3.3$ & 0.503 \\
Total number of resected lymph nodes & $24.3 \pm 3.6$ & $8.7 \pm 1.9$ & 0.206 \\
Thoracic lymph nodes & $5.3 \pm 1.9$ & $18.6 \pm 2.4$ & 0.622 \\
Abdominal lymph nodes & $16.9 \pm 2.5$ & & .
\end{tabular}

Data are presented as the means \pm SEM. ${ }^{a} \mathrm{P}<0.05$ was considered to indicate a statistically significant difference.

Table III. Comparison of postoperative factors between the patients performed middle and lower esophagectomy (anastomosis in thoracic cavity) with and without laparoscopic transhiatal approach (LTHA).

\begin{tabular}{|c|c|c|c|}
\hline \multirow[b]{2}{*}{ Variables } & \multicolumn{2}{|c|}{ LTHA } & \multirow[b]{2}{*}{ P-value } \\
\hline & With & Without & \\
\hline Extubation time after surgery (days) & $0.9 \pm 1.1$ & $4.1 \pm 1.0$ & $0.044^{\mathrm{a}}$ \\
\hline $\begin{array}{l}\text { Postoperative respiratory complications } \\
{[\mathrm{n} / \text { total }(\%)]}\end{array}$ & $1 / 10(10.0)$ & $4 / 11(36.3)$ & 0.311 \\
\hline \multicolumn{4}{|l|}{ White blood cells $(\mathrm{n} / \mu \mathrm{l})$} \\
\hline 2 days after surgery & $9,820 \pm 1,749$ & $12,718 \pm 1,710$ & 0.245 \\
\hline 7 days after surgery & $7,422 \pm 1,204$ & $8,782 \pm 1,106$ & 0.413 \\
\hline \multicolumn{4}{|l|}{ Serum CRP (mg/dl) } \\
\hline 2 days after surgery & $19.1 \pm 2.2$ & $21.7 \pm 2.1$ & 0.397 \\
\hline 7 days after surgery & $7.2 \pm 2.3$ & $6.6 \pm 2.1$ & 0.843 \\
\hline Duration of thoracic drainage (days) & $6.9 \pm 2.9$ & $19.2 \pm 2.7$ & $0.006^{\mathrm{a}}$ \\
\hline Anastomotic leakage (cases/total) & $1 / 10(10.0 \%)$ & $1 / 11(9.1 \%)$ & 1.000 \\
\hline Postoperative hospital stay (days) & $25.7 \pm 5.3$ & $40.7 \pm 5.2$ & $0.025^{\mathrm{a}}$ \\
\hline
\end{tabular}

Data are presented as the means $\pm \mathrm{SEM}$. ${ }^{\mathrm{a}} \mathrm{P}<0.05$ was considered to indicate a statistically significant difference. $\mathrm{CRP}, \mathrm{C}$-reactive protein.

The extubation time after surgery was significantly shorter in the patients treated with compared to that in patients treated without LTHA (mean \pm SEM: $0.9 \pm 1.1$ vs. $4.1 \pm 1.0$ days, respectively). Postoperative respiratory complications occurred in $10.0 \%$ of the patients treated with the LTHA (pneumonia in 1 patient), whereas they occurred in $36.3 \%$ of the patients treated without LTHA (pneumonia in 4 patients), without a statistically significant difference. We analyzed the number of peripheral white blood cells and the serum CRP level at 2 and 7 days after the operation. Although the elevation in the number of peripheral white blood cells at 2 days after the operation tended to be more limited in patients treated with compared to that in patients treated without LTHA (mean \pm SEM: $9,820 \pm 1,749$ vs. $12,718 \pm 1,710 / \mu 1$, respectively), the differences were not considered to be significant. The duration of thoracic drainage was significantly shortened in patients treated with compared to those treated without
LTHA (mean \pm SEM: $6.9 \pm 2.9$ vs. 19.2 \pm 2.7 days, respectively), suggesting that this method decreased the amount of postoperative thoracic discharge by preventing damage to the thoracic and mediastinal tissues. The frequency of anastomotic leakage did not differ significantly between the two groups. The postoperative hospital stay was significantly shortened in patients treated with compared to those treated without LTHA (mean \pm SEM: $25.7 \pm 5.3$ vs. $40.7 \pm 5.2$ days, respectively). These results suggest that LTHA-preceded esophagectomy may be used to improve postoperative care without increasing the risk of major complications.

\section{Discussion}

The surgical trauma caused by esophagectomy is greater compared to that caused by any other general surgical operations. Therefore, pulmonary complications remain the most common 
and serious complications following esophagectomy (2-4). It was recently demonstrated that thoracoscopic (15-19) or mediastinoscopic esophagectomy $(20,21)$ minimizes injury to the chest wall and reduces surgical invasiveness. Furthermore, the duration of one-lung ventilation is known to affect postoperative immune reactions and cause respiratory complications $(6,7)$. We were able to perform middle and lower thoracic mediastinal surgery from the abdominal side of the diaphragm via LTHA and the subsequent thoracic operations, which were performed via a right thoracotomy, were markedly simplified. Therefore, the thoracic trauma was minimized and the duration of one-lung ventilation was reduced. Furthermore, our results demonstrated that the total intraoperative blood loss was significantly decreased and the duration of thoracic drainage was significantly shortened using LTHA. We consider that one of the reasons is that this method enables dissection of the appropriate layer under a magnified videoscopic view and, therefore, damage to the microvessels and lymphatic ducts is avoided $(8,9)$. In our experience, there were no problems associated with applying a $10-\mathrm{mmHg}$ pneumomediastinum pressure for intraoperative respiratory care. All these factors may contribute to improved postoperative outcome, regarding the extubation time following surgery and the postoperative hospital stay.

An additional advantage of this surgical procedure is that it enables easy access to the posterior mediastinal lymph nodes, including the paraaortic and left pulmonary ligament lymph nodes (9). The left side of the mediastinum is a difficult space to approach via a right thoracotomy. In particular, complete resection of the left pulmonary ligament lymph nodes via a right thoracic approach carries an increased risk of serious complications. Furthermore, when performing thoracoscopic esophagectomy in the left lateral decubitus position, it is difficult to maintain the surgical field in the lower mediastinum (15-17). With this method, the gas supply during dissection of the mediastinum makes the layers between the esophagus and the surrounding organs easy to identify and the separation of these layers helps minimize bleeding. Accordingly, the separation of the anterior and posterior sides of the posterior mediastinal lymph nodes is easy to perform and, by lifting up these lymph nodes like a membrane, the border of the left mediastinal pleura may be clearly identified, enabling en-bloc dissection (9). Furthermore, by exposing the adventitia of the thoracic aorta and the crura of the diaphragm, the appropriate layer for the dissection of the celiac lymph nodes may be clearly identified and the lymph nodes along the celiac artery may be dissected continuously.

We previously demonstrated the efficacy of LTHA-preceded subtotal esophagectomy with gastric tube reconstruction via the retrosternal route with regard to the perioperative outcomes of patients with esophageal cancer (8). Namely, LTHA-preceded esophagectomy shortened the intrathoracic operative time, decreased intraoperative bleeding and reduced the amount of postoperative thoracic discharge, without increasing the risk of major postoperative complications (8). In the present study, similar tendencies were observed in patients treated with LTHA-preceded middle and lower esophagectomy. In both studies, the elevation in the number of peripheral white blood cells 2 days after the operation tended to be more limited in patients treated with LTHA, suggesting that this method downregulated excessive immune responses (8). As previously demonstrated, we performed operations using HALS to avoid injury of the reconstructed organ (8-10). The abdominal and the mediastinal operation may be easily performed and, thus far, there has been no report of reconstructed organs suffering intraoperative injuries. Furthermore, we have mainly been using the EnSeal device to avoid damaging the surrounding organs (8-10). The tip of the EnSeal blade is not too sharp and is therefore suitable for safe dissection. In the present study, the same procedure was used for distal esophageal cancer and the extubation time following surgery and postoperative hospital stay were significantly shortened, suggesting that the advantages of the LTHA-preceded method are more distinct in middle and lower esophagectomy.

In conclusion, LTHA-preceded middle and lower esophagectomy, significantly shortened the total operative time and the duration of one-lung ventilation and decreased intraoperative blood loss. Furthermore, the extubation time after surgery, the duration of thoracic drainage and postoperative hospital stay were significantly shortened. Additionally, this procedure ensured a good surgical view of the posterior and left mediastinum, enabling safe en bloc lymph node dissection around these areas, without increasing the risk of major perioperative complications.

\section{References}

1. Tachibana M, Kinugasa S, Yoshimura H, et al: Clinical outcomes of extended esophagectomy with three-field lymph node dissection for esophageal squamous cell carcinoma. Am J Surg 189: 98-109, 2005.

2. Whooley BP, Law S, Murthy SC, Alexandrou A and Wong J: Analysis of reduced death and complication rates after esophageal resection. Ann Surg 233: 338-344, 2001.

3. Law S, Wong KH, Kwok KF, Chu KM and Wong J: Predictive factors for postoperative pulmonary complications and mortality after esophagectomy for cancer. Ann Surg 240: 791-800, 2004

4. Ferguson MK and Durkin AE: Preoperative prediction of the risk of pulmonary complications after esophagectomy for cancer. J Thorac Cardiovasc Surg 123: 661-669, 2002.

5. Bailey SH, Bull DA, Harpole DH, et al: Outcomes after esophagectomy: a ten-year prospective cohort. Ann Thorac Surg 75: 217-222, 2003.

6. Zingg U, Forberger J, Frey DM, et al: Inflammatory response in ventilated left and collapsed right lungs, serum and pleural fluid, in transthoracic esophagectomy for cancer. Eur Cytokine Netw 21: 50-57, 2010.

7. De Conno E, Steurer MP, Wittlinger M, et al: Anesthetic-induced improvement of the inflammatory response to one-lung ventilation. Anesthesiology 110: 1316-1326, 2009.

8. Shiozaki A, Fujiwara H, Murayama Y, et al: Perioperative outcomes of esophagectomy preceded by the laparoscopic transhiatal approach for esophageal cancer. Dis Esophagus: Oct 22, 2012 (Epub ahead of print).

9. Shiozaki A, Fujiwara H, Murayama Y, et al: Posterior mediastinal lymph node dissection using the pneumomediastinum method for esophageal cancer. Esophagus 9: 58-64, 2012.

10. Shiozaki A, Fujiwara H, Daisuke I, Okamoto K, Komatsu S and Otsuji E: Pneumomediastinum method for esophageal cancer. Operation 65: 1277-1280, 2011.

11. Shiozaki A, Fujiwara H, Murayama Y, et al: Hand-assisted laparoscopic transhiatal approach for mediastinal esophageal duplication cyst resection. Esophagus 9: 247-251, 2012.

12. Sobin L, Gospodarowicz M, Wittekind C (eds). TNM Classification of malignant tumors. 7th edition. John Wiley \& Sons, Inc., Hoboken, NJ, 2009.

13. Ando N, Kato $\mathrm{H}$, Igaki $\mathrm{H}$, et al: A randomized trial comparing postoperative adjuvant chemotherapy with cisplatin and 5-fluorouracil versus preoperative chemotherapy for localized advanced squamous cell carcinoma of the thoracic esophagus (JCOG9907). Ann Surg Oncol 19: 68-74, 2012. 
14. Charlson ME, Pompei P, Ales KL and MacKenzie CR: A new method of classifying prognostic comorbidity in longitudinal studies: development and validation. J Chron Dis 40: 373-383, 1987.

15. Osugi H, Takemura M, Lee S, et al: Thoracoscopic esophagectomy for intrathoracic esophageal cancer. Ann Thorac Cardiovasc Surg 11: 221-227, 2005.

16. Osugi H, Takemura M, Higashino M, Takada N, Lee S and Kinoshita H: A comparison of video-assisted thoracoscopic oesophagectomy and radical lymph node dissection for squamous cell cancer of the oesophagus with open operation. Br J Surg 90: 108-113, 2003.

17. Thomson IG, Smithers BM, Gotley DC, et al: Thoracoscopic-assisted esophagectomy for esophageal cancer: analysis of patterns and prognostic factors for recurrence. Ann Surg 252: 281-291, 2010.
18. Noshiro H, Iwasaki H, Kobayashi K, et al: Lymphadenectomy along the left recurrent laryngeal nerve by a minimally invasive esophagectomy in the prone position for thoracic esophageal cancer. Surg Endosc 24: 2965-2973, 2010.

19. Cadière GB, Dapri G, Himpens J and Rajan A: Thoracoscopic esophagectomy in prone position. Ann Surg Oncol 18: 838, 2011.

20. Tangoku A, Yoshino S, Abe T, et al: Mediastinoscope-assisted transhiatal esophagectomy for esophageal cancer. Surg Endosc 18: 383-389, 2004.

21. Mimatsu K, Oida T, Kawasaki A, et al: Mediastinoscopy-assisted esophagectomy is useful technique for poor surgical-risk patients with thoracic esophageal cancer. Surg Laparosc Endosc Percutan Tech 19: e17-e20, 2009. 